\title{
Should the FDA be the doctor of last resort?
}

\author{
Vincent T DeVita, Jr
}

In this issue of the journal, Seeber and Braun highlight that, after FDA approval, new drugs are not made available to patients other than in ways approved and dictated by specific trials. Trial design is often flawed by restricted access to investigational drugs in the control arm of these studies. The FDA, by implication, has become the oncologist of last resort, overriding the training and experience of practicing physicians.

In the past, once a drug was approved by the FDA, its use in combination was not further regulated by the agency. Thus, some of the most famous and durable (and curative) drug combinations, used in patients with lymphomas, leukemias and breast cancer, were developed in post-approval, nonrandomized, non-investigational-new-drug studies. These combinations were never specifically approved by the FDA. This accounts for their rapid development and dissemination, with a notable impact on national mortality rates.

Cisplatin represents a model example of the impact of such tight regulation on clinical practice. In the early 1980s almost every oncologist knew that cisplatin was a powerful addition to other drug combinations used to treat metastatic testicular cancer. In investigational studies, its use, in combination, appeared to increase the complete remission rates well over those achieved with older combinations. Nonetheless, the FDA delayed approval of cisplatin for 3 years, because single-drug efficacy in testicular cancer was not demonstratedeven though testing of cisplatin alone would have exposed patients to noncurative treatment. In other words, experienced investigators had immediately used the drug for combination chemotherapy because combined chemotherapy was required to produce complete remission in metastatic testicular cancer and no one has ever been cured
The FDA, by

implication,

has become

the oncologist

of last resort, overriding the

training and

experience

of practicing

physicians.

VT DeVita, Jr is

the Editor-in-Chief

of Nature Clinical

Practice Oncology.

Competing interests

The author declared he has

competing interests; go to

the article online for details.

www.nature.com/clinicalpractice doi:10.1038/ncponc0292 without complete remission. The Department of Health and Human Services, encouraged by the US National Cancer Institute, increased the patent life of the drug by 3 years to compensate Bristol-Myers once this egregious error surfaced. The impression that cisplatin was only useful in testicular cancer was negated by post-marketing, non-investigational-new-drug trials in other tumors, where it has become a mainstay of treatment.

In the US, regulation of new cancer drugs is governed by the Federal Food, Drug, and Cosmetic (FDC) Act of 1938 and the Kefaufer amendments of 1962. The 1938 Act required the FDA to assure safety of any new drug that was to be marketed. In 1962, the Kefaufer amendments modified this to include the responsibility for determining "substantial efficacy".

Neither of these Acts of the US Congress required the current practice of approval of each new drug on a trial-by-trial basis, nor did they require randomized controlled trials; just "adequately" controlled trials. In recent years, the FDA, presumably with the concurrence of the oncology community, has assumed the authority to limit both the ability to develop new drugs and the development of ideal ways to use these drugs in post-marketing studies. Even 'efficacy' has become a problematic definition. With targeted therapies, the more specific a drug is against its target, the less efficacy it has alone (with a few notable exceptions), leading to the irony that the 'efficacy' rule, as it now stands, may well impede development.

It is unlikely that FDA staff will have a sufficient variety of experience to accommodate the nuances involved in the practice of oncologic medicine today, and limiting use of drugs to study-related uses creates a stultifying regulatory environment. This is occurring at a point in the history of cancer medicine when we need more, not less, flexibility. 